\title{
Orbital maneuver optimization using time-explicit power series
}

\author{
James D. Thorne
}

Received: 23 September 2009 / Revised: 10 November 2010 / Accepted: 15 February 2011 /

Published online: 13 March 2011

(C) The Author(s) 2011. This article is published with open access at Springerlink.com

\begin{abstract}
Orbital maneuver transfer time optimization is traditionally accomplished using direct numerical sampling to find the mission design with the lowest delta-v requirements. The availability of explicit time series solutions to the Lambert orbit determination problem allows for the total delta-v of a series of orbital maneuvers to be expressed as an algebraic function of only the individual transfer times. The delta-v function is then minimized for a series of maneuvers by finding the optimal transfer times for each orbital arc. Results are shown for the classical example of the Hohmann transfer, a noncoplanar transfer as well as an interplanetary fly-by mission to the asteroids Pallas and Juno.
\end{abstract}

Keywords Orbital maneuver optimization · Lambert problem ·

Orbital mission planning - Thorne series solution of lambert problem

\section{Introduction}

Orbital maneuver optimization as a function of the transfer time is traditionally accomplished using either classical calculus of variations techniques for restricted cases, or by direct numerical sampling to minimize the magnitude of the required changes in velocity vectors $(\Delta v)$. However, numerical sampling will not guarantee optimality and can offer only limited insight into the qualitative behavior of the system either inside or outside of the assigned search space.

To find the required velocity components before and after each maneuver point with specified transfer times, one must somehow solve the Lambert orbit determination problem for each transfer arc. Lambert's Theorem states that there is unique value of the semi-major axis, $a$, associated with the arc of a single conic section that will correspond to a given flight time, $t$, and the given problem geometry. Solving the Lambert orbit determination problem amounts to finding $a$ such that all of the given conditions are satisfied. Once the unknown

J. D. Thorne $(\varangle)$

System Evaluation Division, Institute for Defense Analyses, Alexandria, VA 22311, USA

e-mail: jthorne@ida.org 
$a$ has been found, the orbit is completely determined and the velocity components may be calculated at any point.

The Lambert problem may be expressed in terms of the Lagrange trajectory equations, which equate the transfer time $t$ to transcendental functions of the unknown semi-major axis. However, because the functional dependence of these equations is the opposite of what would be desired, some form of numerical solution is normally required to solve them. Many robust root-finding techniques (Battin 1987; Gooding 1990) have been developed to solve the Lambert problem. Some of these methods have very good convergence properties over large ranges of the transfer angle and distance ratios. In all cases, though, these methods employ iterative root-finding, and do not provide an analytical, explicit solution for $a$ as a function of $t$. Without an explicit solution for $a$ as a function of $t$, it is not possible to set up an analytical framework to optimize a series of maneuvers to find the miminum total $\Delta v$. In this paper, the goal is to demonstrate the ability to find such analytical minima.

In addition, other investigators have examined the Lambert Problem through expansions about a numerical solution (Lizia et al. 2008), or solved it for conjunction analysis (Peterson 2002) or in light of gravitational perturbations (Park et al. 1998), but these analyses are each focused on a single case of orbital geometry at a time rather than the simultaneous optimization of a sequence of individual maneuvers.

Recently, time series solutions have been found (Thorne 2004; Vallado 2007) to solve all orbital cases of the Lambert problem by analytically reversing the functional dependence of the Lagrange trajectory equations from $a$ to $t$. The availability of the complete set of time series solutions for the Lambert orbit determination problem allows for the total $\Delta v$ magnitude for a series of orbital maneuvers to be written as a single algebraic expression, an explicit function of only the individual transfer times. By truncating the resulting power series terms to achieve the desired accuracy, these solutions may be used as polynomial functions for the unknown $a$ of each orbital transfer arc. Using classical calculus techniques, the $\Delta v$ function may then be minimized for a series of sequential maneuvers by finding the set of optimal flight times for each of the individual orbital transfer arcs. Other traditional iterative methods for solving the Lambert Problem may be more compact or numerically robust for certain parameter spaces (although they may not converge at all in some cases), but they cannot be used as part of an analytical technique for maneuver optimization since all of them involve root-finding methods rather than explicit expressions. Thus, the point of this paper is not to compare computational speed or accuracy of the various traditional means with the time-explicit power series solutions of the Lambert Problem, but to exploit these series solutions (Thorne 2004; Vallado 2007) of the Lagrange trajectory equations for analytical optimization which would not be possible otherwise.

The basic problem geometry is shown in Fig. 1 below, where $c_{n}$ is the $n$th chord line between position vectors $r_{n}$ and $r_{n+1}$. The semiperimeter, $s_{n}$, is defined by $s_{n}=$ $\left(r_{n}+r_{n+1}+c_{n}\right) / 2$.

\section{Analytical methodology}

The total change in velocity is the sum of $n$ vector differences. The goal is to minimize the objective function, $J$, which is the sum of the magnitudes of the total number of the velocity changes in the orbital mission. 
Fig. 1 Multiple-transfer Lambert problem geometry

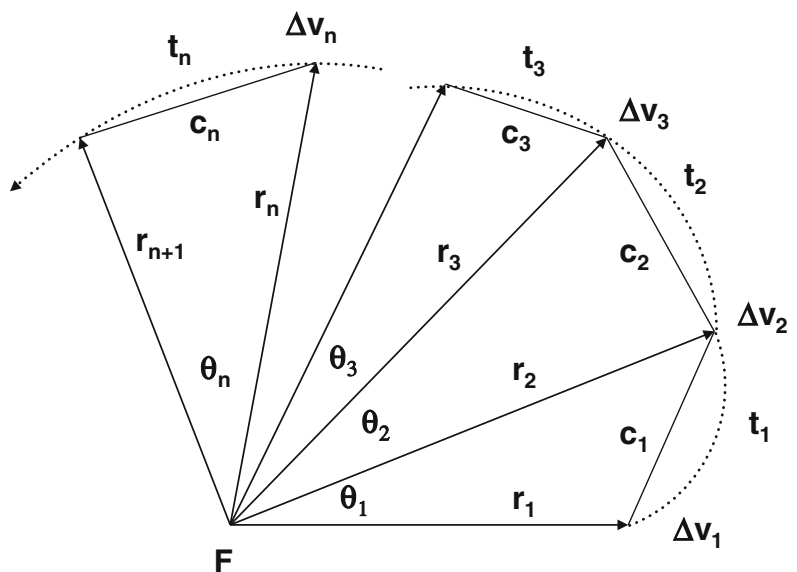

$$
S_{n}=\left(r_{n}+r_{n+1}+c_{n}\right) / 2
$$

$$
\begin{aligned}
J=\sum_{n=1}^{\text {total }} \Delta v_{n}= & \sqrt{\left(\dot{x}_{1}-\dot{x}_{0}\right)^{2}+\left(\dot{y}_{1}-\dot{y}_{0}\right)^{2}+\left(\dot{z}_{1}-\dot{z}_{0}\right)^{2}}+\cdots \\
& +\sqrt{\left(\dot{x}_{2 n-1}-\dot{x}_{2 n-2}\right)^{2}+\left(\dot{y}_{2 n-1}-\dot{y}_{2 n-2}\right)^{2}+\left(\dot{z}_{2 n-1}-\dot{z}_{2 n-2}\right)^{2}}
\end{aligned}
$$

One way to find the velocity at the $n$th point in the orbit is to use the Lagrange coefficient functions $f, g$ and their time derivatives (Battin 1987). Here is an example using the two Cartesian coordinates, $x$ and $y$ :

For $n$ maneuvers, the velocity components are:

$$
\begin{aligned}
\dot{x}_{2 n-1} & =\frac{1}{g_{n}} x_{n+1}-\frac{f_{n}}{g_{n}} x_{n} \\
\dot{y}_{2 n-1} & =\frac{1}{g_{n}} y_{n+1}-\frac{f_{n}}{g_{n}} y_{n} \\
\dot{x}_{2 n} & =\dot{f}_{n} x_{n}+\dot{g}_{n} \dot{x}_{2 n-1} \\
\dot{y}_{2 n} & =\dot{f}_{n} y_{n}+\dot{g}_{n} \dot{y}_{2 n-1}
\end{aligned}
$$

where the Lagrange coefficient functions $f_{n}, g_{n}, \dot{f}_{n}, \dot{g}_{n}$ are given by:

$$
\begin{aligned}
& f_{n}=1-\frac{a_{n}}{r_{n}}\left(1-\cos \left(\Delta E_{n}\right)\right) \\
& g_{n}=t_{n}-\sqrt{\frac{a_{n}^{3}}{\mu}}\left(\Delta E_{n}-\sin \left(\Delta E_{n}\right)\right) \\
& \dot{f}_{n}=-\frac{\sqrt{\mu a_{n}}}{r_{n} r_{n+1}} \sin \left(\Delta E_{n}\right) \\
& \dot{g}_{n}=1-\frac{a_{n}}{r_{n+1}}\left(1-\cos \left(\Delta E_{n}\right)\right)
\end{aligned}
$$

The change in eccentric anomaly $\Delta E_{n}$ can be found by using the Lagrange parameters $\alpha_{n}$ and $\beta_{n}$, depending on the type of orbit transfer. For arcs of ellipses, the four possibilities are: 
Fig. 2 Example semi-major axis plot, canonical units, $\mu=1$
Semi-Major Axis vs. Time $r_{1}=r_{2}=1$, Transfer Angle $=180 \mathrm{deg}$

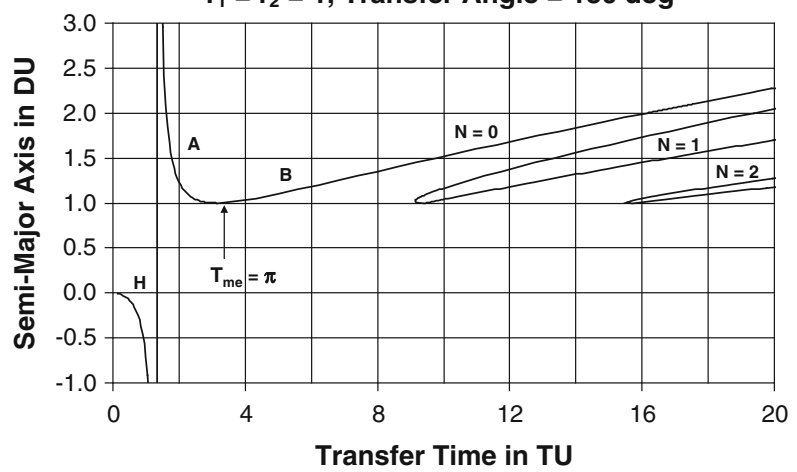

\begin{tabular}{|l|l|}
\hline 1A: $\left(\theta<180^{\circ}\right.$, short way $)$ & $\Delta E_{n}=\alpha_{n}-\beta_{n}$ \\
\hline 1B: $\left(\theta<180^{\circ}\right.$, long way $)$ & $\Delta E_{n}=2 \pi-\alpha_{n}-\beta_{n}$ \\
\hline 2A: $\left(\theta>180^{\circ}\right.$, short way $)$ & $\Delta E_{n}=\alpha_{n}+\beta_{n}$ \\
\hline 2B: $\left(\theta>180^{\circ}\right.$, long way $)$ & $\Delta E_{n}=2 \pi-\alpha_{n}+\beta_{n}$ \\
\hline
\end{tabular}

The Lagrange parameters are defined as follows (Battin 1987):

$$
\alpha_{n}=2 \sin ^{-1} \sqrt{\frac{s_{n}}{2 a_{n}}}, \quad \beta_{n}=2 \sin ^{-1} \sqrt{\frac{s_{n}-c_{n}}{2 a_{n}}}
$$

where $a_{n}$ is the semi-major axis of the $n$th transfer orbit and $\mu$ is the gravitational constant. The hyperbolic cases are similar. The specific type of orbit may be determined unambiguously by considering the given problem geometry, the number of orbital revolutions $(N)$ and the range of transfer times. In accordance with the Lambert Theorem, there is only one possible transfer arc associated with one conic section that will satisfy the given conditions of the problem once the transfer time is specified. The various orbit transfer types are indicated in Fig. 2, which shows the relationship between $a$ and $t$ for a representative example geometry.

It has been shown previously (Thorne 2004) that the series solution for both the hyperbolic and short-way elliptic transfer type (A) is given by:

$$
a=\left(\frac{s}{2}\right) \sum_{k=0}^{\infty} B_{k}\left(\frac{t}{t_{p}}-1\right)^{(k-1)}
$$

where $t_{p}$ is the parabolic transfer time for the given geometry and the $\mp$ signs correspond to transfer angles of less than or greater than $180^{\circ}$, respectively:

$$
\text { 1P, 2P: } \quad t_{p}=\frac{1}{3} \sqrt{\frac{2 s^{3}}{\mu}}\left[1 \mp\left(\frac{s-c}{s}\right)^{3 / 2}\right]
$$

The series solution for long-way transfers (B) near the minimum-energy time is given by (Thorne 2004):

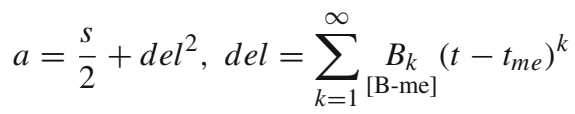

The solution for the long-way elliptic case (B) as the transfer time approaches infinity is (Thorne 2004): 


$$
a=\sum_{k=0}^{\infty} \underset{[\mathrm{B}-\mathrm{inf}]}{B_{k}} t^{\left(\frac{2-k}{3}\right)}
$$

The series solutions given in Eqs. (12) through (15) may be used to produce all of the data shown in Fig. 2, including the multiple-revolution cases. Although all of the $B_{k}$ coefficients in these series equations are functions of the problem geometry, they differ by orbit transfer type (Thorne 2004).

As defined in Eq. (1), $J$ is a function of the velocity vector, $\vec{v}$, which is the first time derivative of $\vec{r}$.

$$
J=J(\vec{v})
$$

The velocity components are functions of the $f$ and $g$ expressions and their time derivatives, and the components of the position vectors which are given constants. Denoting this group of expressions by $f$, we have:

$$
J=J(\vec{v}(f))
$$

The $f$ and $g$ expressions and their time derivatives are functions of $a_{n}, t_{n}$ and $\Delta E_{n}$, so $J$ becomes:

$$
J=J\left(\vec{v}\left(f\left(a_{n}, t_{n}, \Delta E_{n}\right)\right)\right)
$$

Finally, $\Delta E_{n}$ is a function of $a_{n}$, and $a_{n}$ is a time series in $t_{n}$, so $J$ can be expressed as a single algebraic function of $t_{n}$ as follows:

$$
J=J\left(\vec{v}\left(f\left(\Delta E_{n}\left(a_{n}\left(t_{n}\right)\right)\right)\right)\right)
$$

Starting with Eq. (1), one may substitute Eqs. (2) through (15) as appropriate into $J$ to produce an algebraic function of only the transfer times, $t_{n}$, and known constants. The $J$ function also contains no trigonometric terms, so there are no issues of quadrant checks, etc. This repeated substitution process can produce a very lengthy expression which may be evaluated using a symbolic manipulation software package. In spite of the resulting length, however, $J$ may be minimized with respect to the $t_{n}$ with little difficulty. In the following two examples, $J$ is minimized for a classical Hohmann transfer problem in two dimensions, and also for a flyby mission to two asteroids in three-dimensional space.

Example 1 (Hohmann Transfer) The Hohmann transfer is well known to be the most fuelefficient way to move a satellite from one coplanar circular orbit to another with two impulsive maneuvers. The optimality of the Hohmann transfer can be shown in several ways, including the use of Lagrange multipliers (Battin 1987). However, if more than two maneuvers are required to visit multiple orbital locations, the minimum $\Delta v$ problem can become extremely complicated.

To check the behavior of the objective function $J$ against a well-known optimal solution, the geometry of a typical Hohmann transfer is broken into three transfer arcs as shown in Fig. 3. In this example, all three orbit transfers are of type $1 \mathrm{~A}$, and so the change in eccentric anomaly for all three transfer arcs is given by $\Delta E_{n}=\alpha_{n}-\beta_{n}$.

The geometry of Fig. 3 may be summarized in tabular form as follows:

\begin{tabular}{|l|l|l|l|l|l|l|}
\hline$n$ & $x_{n}$ & $y_{n}$ & $r_{n}$ & $\theta_{n}$ & $c_{n}$ & $s_{n}$ \\
\hline 1 & 1 & 0 & 1 & $\pi / 2$ & 1.8027756 & 2.1513878 \\
2 & 0 & 1.5 & 1.5 & $\pi / 6$ & 1.0265708 & 2.2632854 \\
3 & -1 & $\sqrt{3}$ & 2 & $\pi / 3$ & 2.6457513 & 3.8228757 \\
4 & -3 & 0 & 3 & - & - & - \\
\hline
\end{tabular}


Fig. 3 Hohmann transfer geometry

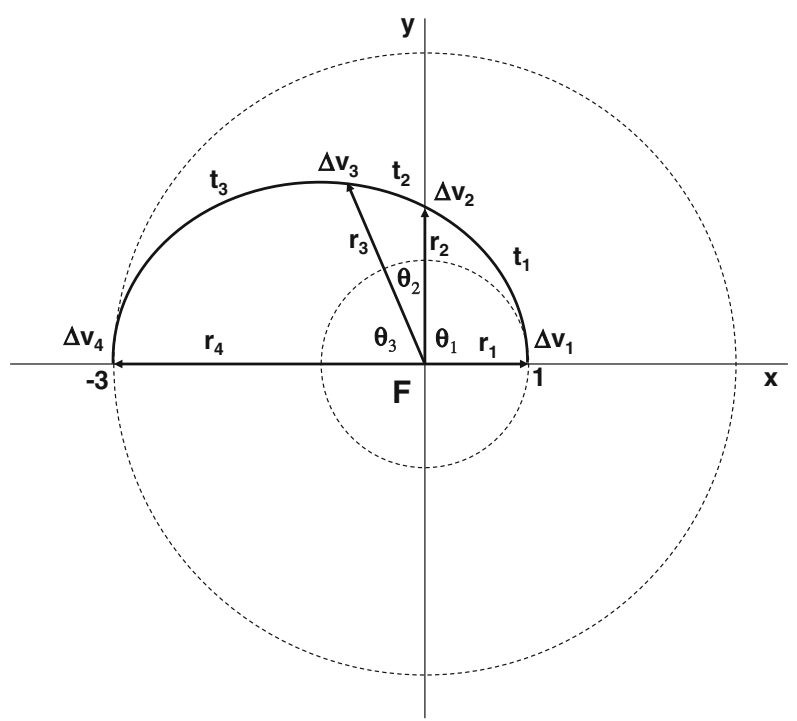

In this example, the gravitational parameter is taken to be $\mu=1$ as part of a set of canonical units. To do the minimization analytically, the unknown semi-major axis of each of the three transfer arcs must be expressed as a time series. The following time series equations contain the numerical values of the first few series coefficients for each of the three unknown semi-major axes for the Hohmann transfer example. The argument of each series is the quantity $T_{n}=\left(t_{n} / t_{p n}-1\right)$ as shown in Eq. (12), where $t_{p n}$ is the parabolic transfer time with respect to the $n$th transfer. The numerical values of the coefficients may be produced from the given problem geometry using recursive relationships (Thorne 2004). The series terms are normally calculated out to the 15th order, which takes well less than one second of calculation time on a modern desktop computer, although fewer terms are shown below in the interest of space. Typically, using this number of terms will result in an accuracy of at least three significant figures and often many more, so that there is no question that the time optimization technique has correctly identified the minimum $\Delta v$ solution.

$$
\begin{aligned}
a_{1}= & 0.341577792 \times T_{1}^{-1}+0.581423045 \times T_{1}^{0}+0.198793408 \times T_{1}^{1}-0.019664019 \times T_{1}^{2} \\
& +0.006827650 \times T_{1}^{3}-0.004289367 \times T_{1}^{4}+0.003389308 \times T_{1}^{5}-0.002685007 \times T_{1}^{6} \\
& +0.001958767 \times T_{1}^{7}-0.001268051 \times T_{1}^{8}+0.000700027 \times T_{1}^{9}+\cdots \\
a_{2}= & 0.443837605 \times T_{2}^{-1}+0.684115989 \times T_{2}^{0}+0.146400733 \times T_{2}^{1}-0.040157343 \times T_{2}^{2} \\
& +0.025632458 \times T_{2}^{3}-0.014076714 \times T_{2}^{4}+0.007073272 \times T_{2}^{5}-0.003479568 \times T_{2}^{6} \\
& +0.001726507 \times T_{2}^{7}-0.000864567 \times T_{2}^{8}+0.000433554 \times T_{2}^{9}+\cdots \\
a_{3}= & 0.655214206 \times T_{3}^{-1}+1.063339971 \times T_{3}^{0}+0.307819469 \times T_{3}^{1}-0.037555013 \times T_{3}^{2} \\
& +0.023112509 \times T_{3}^{3}-0.017028174 \times T_{3}^{4}+0.011051943 \times T_{3}^{5}-0.006153058 \times T_{3}^{6} \\
& +0.002976372 \times T_{3}^{7}-0.001294570 \times T_{3}^{8}+0.000549986 \times T_{3}^{9}+\cdots
\end{aligned}
$$


The series equations for $a_{n}$ may be substituted into the expression for $J$, creating the desired algebraic function in a symbolic manipulator program. The results of minimizing this objective function are as follows, where the exact reference values are shown for comparison to the calculated values:

\begin{tabular}{|l|l|l|l|l|l|l|}
\hline$n$ & $\begin{array}{l}(\mathrm{ref}) t_{n} \\
(\mathrm{TU})\end{array}$ & $\begin{array}{l}(\mathrm{calc}) t_{n} \\
(\mathrm{TU})\end{array}$ & $\begin{array}{l}(\mathrm{ref}) a_{n} \\
(\mathrm{DU})\end{array}$ & $\begin{array}{l}\text { (calc) } a_{n} \\
(\mathrm{DU})\end{array}$ & $\begin{array}{l}\text { (ref) } \Delta v_{n} \\
(\mathrm{DU} / \mathrm{TU})\end{array}$ & $\begin{array}{l}\text { (calc) } \Delta v_{n} \\
(\mathrm{DU} / \mathrm{TU})\end{array}$ \\
\hline & & & & & & \\
1 & 1.73718 & 1.73718 & 2.00000 & 2.00000 & 0.224745 & 0.224745 \\
2 & 1.29149 & 1.29149 & 2.00000 & 2.00000 & 0.000000 & 0.000000 \\
3 & 5.85710 & 5.85968 & 2.00000 & 1.99984 & 0.000000 & 0.0002745 \\
4 & - & - & - & - & 0.169102 & 0.169151 \\
\hline
\end{tabular}

The agreement is quite good between the analytical results and the Hohmann transfer reference case. The differences are a function of the number of series terms that were used in the calculations. As mentioned previously, the accuracy is sufficient to identify the minimum $\Delta v$ case using analytical rather than purely numerical methods by taking advantage of the series solutions. As may be seen above, the optimized second and third $\Delta v$ magnitudes are close to zero, as they should be, since the overall minimum occurs when the maneuvers are exactly the same as the two-impulse Hohmann case.

Example 2 (Noncoplanar Orbit Transfer) The second example was suggested by a presentation in the textbook Fundamentals of Astrodynamics and Applications, 3rd Edition (Vallado 2007) where, in Sect. 6.6 (Ex. 6-10), the problem of a noncoplanar orbital transfer is examined. (The use of a phasing orbit as shown in the textbook reference is not addressed here because it does not affect the total $\Delta v$ for the mission.) In this example the units used are kilometers and seconds, so the gravitational parameter of the earth is taken to be $\mu=$ $398,600 \mathrm{~km}^{3} / \mathrm{s}^{2}$.

Initially, a spacecraft is in a circular parking orbit of radius $7,143 \mathrm{~km}$ and an inclination of $28.5^{\circ}$. After entering a transfer orbit, the final maneuver is used to both circularize the motion at the geosynchronous radius of $42,159 \mathrm{~km}$ and to accomplish the plane change necessary to make the orbit equatorial with zero degrees of inclination. As in the first example of this paper, the transfer is broken into separate arcs to show that the unneeded $\Delta v$ at $r_{2}$ will vanish by virtue of the optimization approach as shown in Fig. 4. The geometry of the problem may be summarized as follows:

\begin{tabular}{|l|l|l|l|l|l|l|l|}
\hline$n$ & $x_{n}$ & $y_{n}$ & $z_{n}$ & $r_{n}$ & $\theta_{n}$ & $c_{n}$ & $s_{n}$ \\
\hline 1 & 7143 & 0 & 0 & 7143 & $\pi / 2$ & 14152 & 16756 \\
2 & 0 & 10737 & 5829 & 12217 & $\pi / 2$ & 43894 & 49135 \\
3 & -42159 & 0 & 0 & 42159 & - & - & - \\
\hline
\end{tabular}

The third manuever includes the plane change. As in Example 1, to do the minimization analytically, the unknown semi-major axis of the two transfer arcs must be expressed as a time series. The following time series equations contain the numerical values of the first few series coefficients for each of the three unknown semi-major axes for the Hohmann transfer example. The argument of each series is the quantity $T_{n}=\left(t_{n} / t_{p n}-1\right)$ as shown in Eq. (12), where $t_{p n}$ is the parabolic transfer time with respect to the $n$th transfer. The numerical values of the coefficients may be produced from the given problem geometry using recursive 
Fig. 4 Non-coplanar Hohmann transfer example geometry

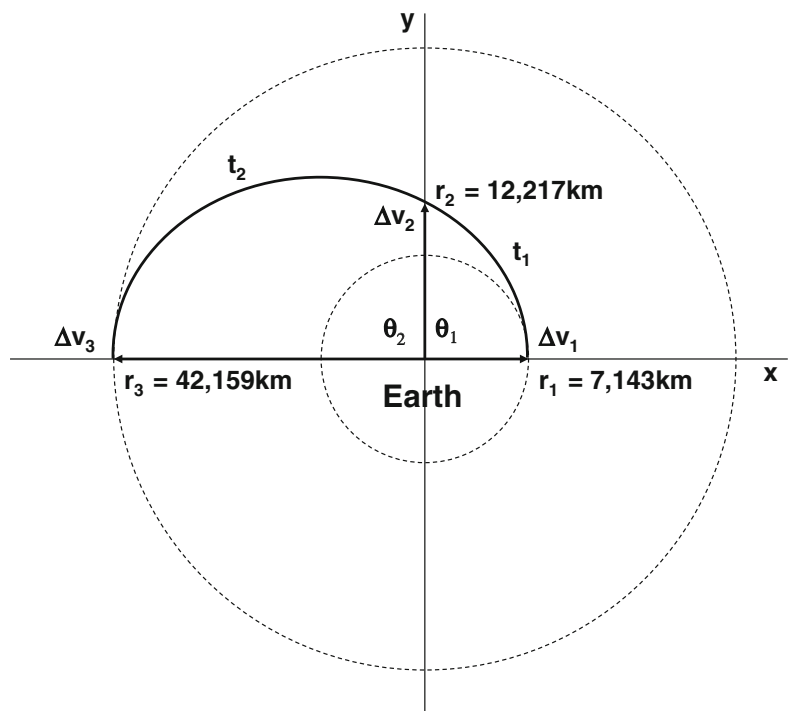

relationships (Thorne 2004) in a manner similar to that shown in Example 1.

$$
\begin{aligned}
a_{1}= & 2652.0 \times T_{1}^{-1}+4524.7 \times T_{1}^{0}+1556.6 \times T_{1}^{1}-154.2 \times T_{1}^{2} \\
& +52.5 \times T_{1}^{3}-31.8 \times T_{1}^{4}+24.9 \times T_{1}^{5}-20.0 \times T_{1}^{6} \\
& +14.9 \times T_{1}^{7}-9.9 \times T_{1}^{8}+5.7 \times T_{1}^{9}+\cdots \\
a_{2}= & 7607.9 \times T_{2}^{-1}+13205.1 \times T_{2}^{0}+4728.1 \times T_{2}^{1}-481.9 \times T_{2}^{2} \\
& +145.3 \times T_{2}^{3}-68.6 \times T_{2}^{4}+45.1 \times T_{2}^{5}-35.7 \times T_{2}^{6} \\
& +29.7 \times T_{2}^{7}-24.0 \times T_{2}^{8}+18.2 \times T_{2}^{9}+\cdots
\end{aligned}
$$

The series equations for $a_{n}$ may be substituted into the expression for $J$, creating the desired algebraic function. The results of minimizing this objective function are as follows, where the exact reference values are shown for comparison to the calculated values:

\begin{tabular}{|l|l|l|l|l|l|l|}
\hline$n$ & $\begin{array}{l}(\mathrm{ref}) t_{n} \\
(\mathrm{sec})\end{array}$ & $\begin{array}{l}(\mathrm{calc}) t_{n} \\
(\mathrm{sec})\end{array}$ & $\begin{array}{l}(\mathrm{ref}) a_{n} \\
(\mathrm{~km})\end{array}$ & $\begin{array}{l}(\mathrm{calc}) a_{n} \\
(\mathrm{~km})\end{array}$ & $\begin{array}{l}(\mathrm{ref}) \Delta v_{n} \\
(\mathrm{~km} / \mathrm{s})\end{array}$ & $\begin{array}{l}(\mathrm{calc}) \Delta v_{n} \\
(\mathrm{~km} / \mathrm{s})\end{array}$ \\
\hline & & & & & & \\
1 & 1722.7 & 1721.1 & 24651.5 & 24813.7 & 2.29887 & $\begin{array}{l}2.30429 \\
1.06965 \times 10^{-7}\end{array}$ \\
2 & 17536.8 & 17562.9 & 24651.5 & 24646.9 & 0 & 1.80245 \\
3 & - & - & - & - & $\begin{array}{l}1.80299 \\
4.107\end{array}$ \\
Totals & 19259.5 & 19284.0 & & & 4.101 & 4.107 \\
\hline
\end{tabular}

As may be seen above, the optimized second $\Delta v$ magnitude is close to zero, as it should be, since the overall minimum occurs when the maneuvers are exactly the same as the Hohmann case with a final plane change. The agreement between the analytical results and the text reference case is close enough to make it clear that the time optimization scheme using 15 series terms successfully identified the minimum $\Delta v$ case, which was the purpose of this example. It is important to notice that the text reference case (Vallado 2007) was only optimized for flight time and not for the inclination of the transfer orbit. However, the optimization for flight time and inclination can be accomplished separately because they are independent of 
Fig. 5 Reference case for Earth-Pallas_-Juno flyby mission in X-Y plane

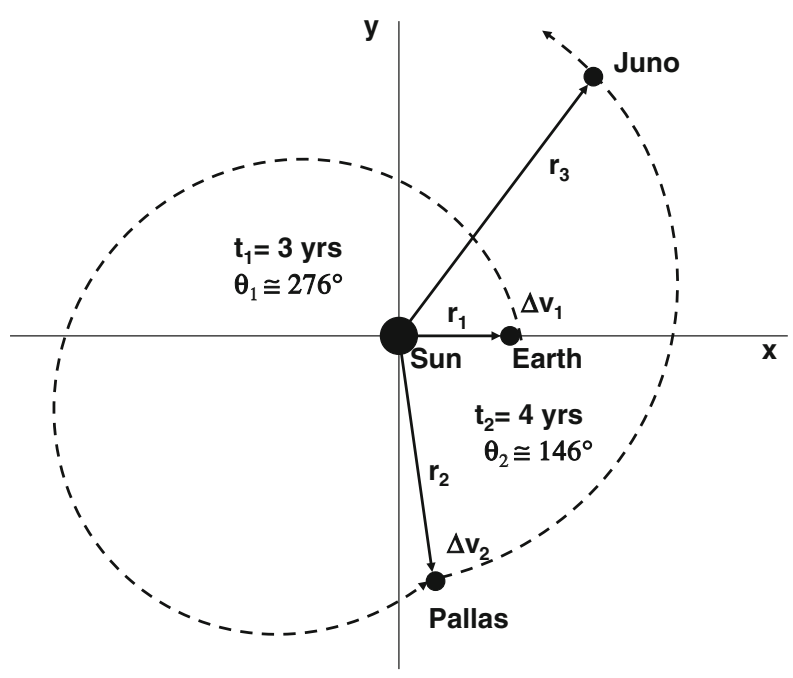

each other. For completeness, the optimal inclination angle of the transfer orbit is $26.16^{\circ}$, where the first inclination change should be $-2.34^{\circ}$ from the parking orbit of $28.5^{\circ}$, with the remaining $-26.16^{\circ}$ to be accomplished with the final maneuver at geosynchronous altitude. By splitting the inclination changes between the two maneuvers in this way, the total $\Delta v$ is reduced from the value of $4.101 \mathrm{~km} / \mathrm{s}$ given in the reference example to $4.074 \mathrm{~km} / \mathrm{s}$. Also, in the text example, the unit conversions between kilometers and earth radii resulted in the truncation of some of the reference values.

Example 3 (Earth-Pallas-Juno Flyby Mission) The third example is taken from a proposed flyby mission to the sun-orbiting asteroids Pallas and Juno, starting from earth parking orbit. The orbits of Pallas and Juno are inclined, but the transfer arcs are shown as projections onto the plane of the ecliptic in Fig. 5.

The initial position vector has a magnitude of one astronomical unit (AU) and the gravitational constant is normalized to unity. This example has an additional constraint that the sum of the two transfer times must be equal to 7 years, with a reference case of $t_{1}=3.0$ years and $t_{2}=4.0$ years. The reference case is shown in Figs. 5 and 6 and it was suggested as part a graduate course project in orbital mechanics taught by Dr. Kathleen C. Howell at Purdue University in 1984 . Because of the constraint that $t_{2}=7-t_{1}$, the only independent variable in the objective function $J$ is $t_{1}$, after substitution.

As before, to do the minimization analytically, the unknown semi-major axis of each of the two transfer arcs must be expressed as a time series. The following equations show the numerical values of the first few coefficients of the series for each of the two unknown semimajor axes and a total of 15 series terms were used as before. Again, the numerical values may be produced from the given problem geometry using recursive relationships (Thorne 2004). To find $a_{1}$ for the first transfer, the argument of the series is, $T_{1}=\left(t_{1} / t_{p 1}-1\right)$ for use in Eq. (12). 
Fig. 6 Reference case for Earth-Pallas_-Juno flyby mission in 3D

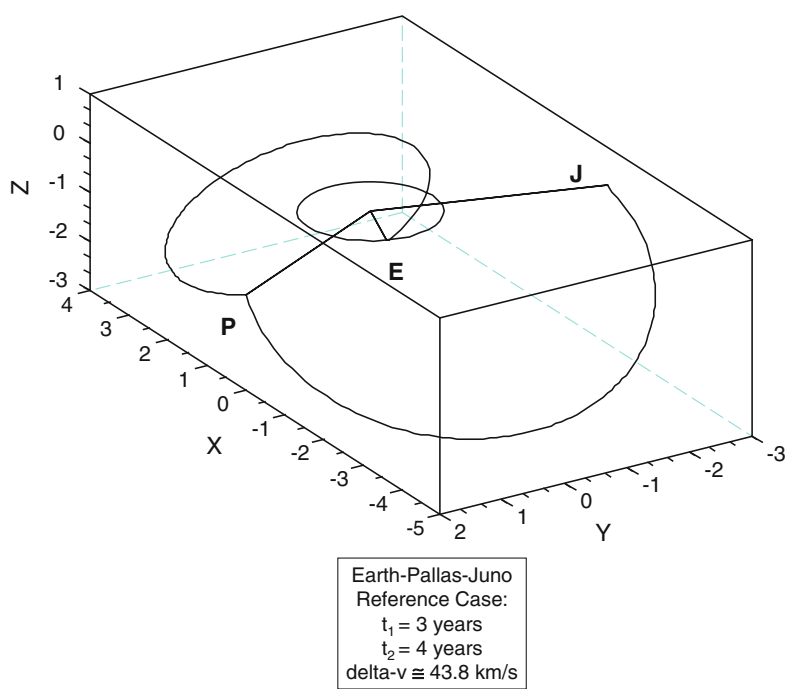

$$
\begin{aligned}
a_{1}= & 0.606326779 \times T_{1}^{-1}+1.082815477 \times T_{1}^{0}+0.405850843 \times T_{1}^{1}-0.044185431 \times T_{1}^{2} \\
& +0.013667178 \times T_{1}^{3}-0.005757163 \times T_{1}^{4}+0.002842438 \times T_{1}^{5}-0.001549493 \times T_{1}^{6} \\
& +0.000904783 \times T_{1}^{7}-0.000556037 \times T_{1}^{8}+0.000355637 \times T_{1}^{9}+\cdots
\end{aligned}
$$

For $a_{2}$, the series solution is given by Eq. (15).

$$
\begin{aligned}
a_{2}= & 0.293683865 \times t_{2}^{(2 / 3)}+0.0 \times t_{2}^{(1 / 3)}+0.0 \times t_{2}^{(0 / 3)}+1.335329741 \times t_{2}^{(-1 / 3)} \\
& +0.0 \times t_{2}^{(-2 / 3)}+4.166086492 \times t_{2}^{(-3 / 3)}-1.517878345 \times t_{2}^{(-4 / 3)} \\
& +22.854605878 \times t_{2}^{(-5 / 3)}-28.413712080 \times t_{2}^{(-6 / 3)}+133.130239337 \times t_{2}^{(-7 / 3)} \\
& -333.662978219 \times t_{2}^{(-8 / 3)}+\cdots
\end{aligned}
$$

\begin{tabular}{|c|c|c|c|c|c|c|c|c|}
\hline $\begin{array}{l}t_{1} \\
\text { (yr) }\end{array}$ & $\begin{array}{l}t_{2} \\
(\mathrm{yr})\end{array}$ & $\begin{array}{l}a_{1} \\
(\mathrm{AU})\end{array}$ & $\begin{array}{l}a_{2} \\
(\mathrm{AU})\end{array}$ & $\begin{array}{l}\theta_{1} \\
(\mathrm{deg})\end{array}$ & $\begin{array}{l}\theta_{2} \\
(\mathrm{deg})\end{array}$ & $\begin{array}{l}\Delta v_{1} \\
(\mathrm{~km} / \mathrm{s})\end{array}$ & $\begin{array}{l}\Delta v_{2} \\
(\mathrm{~km} / \mathrm{s})\end{array}$ & $\begin{array}{l}\Delta v_{\text {total }} \\
(\mathrm{km} / \mathrm{s})\end{array}$ \\
\hline 6.0 & 1.0 & 3.6106 & -7.2124 & 155.185 & 270.740 & 25.8717 & 12.1649 & 38.0366 \\
\hline 5.5 & 1.5 & 3.4315 & 3.2060 & 130.480 & 294.425 & 25.1729 & 4.3954 & 29.5683 \\
\hline 5.0 & 2.0 & 3.2160 & 2.2451 & 104.700 & 318.169 & 27.2576 & 9.3622 & 36.6198 \\
\hline 4.5 & 2.5 & 2.9677 & 2.1211 & 74.564 & 339.899 & 30.9650 & 15.1582 & 46.1232 \\
\hline 4.0 & 3.0 & 2.7029 & 2.5428 & 34.932 & 36.521 & 36.4773 & 37.3787 & 73.8560 \\
\hline 3.5 & 3.5 & 2.4015 & 2.8184 & 338.189 & 87.799 & 33.9103 & 37.4065 & 71.3168 \\
\hline 3.0 & 4.0 & 2.2725 & 3.1339 & 275.896 & 145.733 & 17.9074 & 25.8870 & 43.7944 \\
\hline 2.5 & 4.5 & 2.1861 & 3.3877 & 226.274 & 207.419 & 13.5830 & 16.8808 & 30.4638 \\
\hline 2.0 & 5.0 & 2.1309 & 3.5532 & 191.604 & 238.375 & 5.7391 & 8.9513 & 14.6904 \\
\hline 1.5 & 5.5 & 2.1549 & 3.6537 & 161.016 & 265.005 & 23.9916 & 10.4513 & 34.4429 \\
\hline 1.0 & 6.0 & 2.6540 & 3.7124 & 136.250 & 288.953 & 19.3632 & 11.0029 & 30.3661 \\
\hline
\end{tabular}

where $t_{2}=7-t_{1}$. In canonical units, 1 year is equal to $2 \pi$ time units, so the constraint becomes $t_{2}=14 \pi-t_{1}$.

Here are a range of numerical results, followed by the analytical results for the reference case and an optimized case:

The two highlighted entries show the reference and minimum $\Delta v$ cases using normal propagation of the orbits of Pallas and Juno. However, these results can be improved by 
Fig. 7 Total delta-v versus $\mathrm{t} 1$ for Earth-Pallas-Juno example
Cost Function, J (Earth-Pallas-Juno)

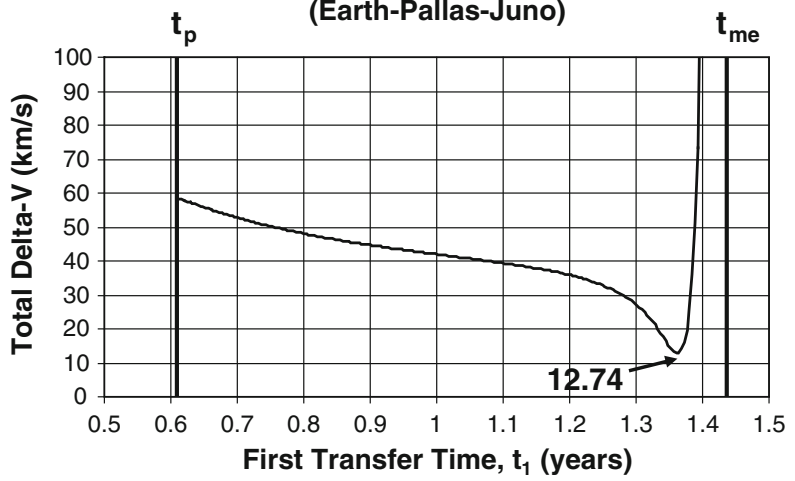

fixing the geometry and using the analytical optimization technique, based on the time series solutions for semi-major axis as seen in the next table. Once the solutions are found for the two fixed geometry examples highlighted in the table, an appropriate initial launch time would need to be calculated such that Pallas and Juno would be in the correct positions to achieve the time-optimal $\Delta v$ values shown below.

\begin{tabular}{|l|l|l|l|l|l|l|l|l|}
\hline $\begin{array}{l}t_{1} \\
(\mathrm{yr})\end{array}$ & $\begin{array}{l}t_{2} \\
(\mathrm{yr})\end{array}$ & $\begin{array}{l}a_{1} \\
(\mathrm{AU})\end{array}$ & $\begin{array}{l}a_{2} \\
(\mathrm{AU})\end{array}$ & $\begin{array}{l}\theta_{1} \\
(\mathrm{deg})\end{array}$ & $\begin{array}{l}\theta_{2} \\
(\mathrm{deg})\end{array}$ & $\begin{array}{l}\Delta v_{1} \\
(\mathrm{~km} / \mathrm{s})\end{array}$ & $\begin{array}{l}\Delta v_{2} \\
(\mathrm{~km} / \mathrm{s})\end{array}$ & $\begin{array}{l}\Delta v_{\text {total }} \\
(\mathrm{km} / \mathrm{s})\end{array}$ \\
\hline & & & & & & & & \\
0.81 & 6.19 & 1.4276 & 3.8356 & $\mathbf{2 7 5 . 8 9 6}$ & $\mathbf{1 4 5 . 7 3 3}$ & 16.3429 & 18.2371 & $\mathbf{3 4 . 5 8 0 0}$ \\
1.36 & 5.64 & 2.0258 & 3.74787 & $\mathbf{1 9 1 . 6 0 4}$ & $\mathbf{2 3 8 . 3 7 5}$ & 4.76238 & 7.97585 & $\mathbf{1 2 . 7 3 8 2}$ \\
\hline
\end{tabular}

The two entries above show the optimized results for fixed geometries with the times allowed to vary independently for the reference and low $\Delta v$ cases. The first case is shown to illustrate the potential improvement over the original reference case, and the second is to take advantage of the lowest $\Delta v$ case found from the full survey of flight times.

Figure 7 is a graph of the behavior of the cost function $J$ in the neighborhood of the minimum for the low $\Delta v$ case, $12.7382 \mathrm{~km} / \mathrm{s}$. For the fixed geometry, the range of flight times indicated between the parabolic transfer time, $t_{p}$, and the minimum energy time, $t_{m e}$, is also the range of validity for the series solution given by Eq. (12) for a 2A-type elliptic transfer.

Figure 8 shows the trajectories for the lowest $\Delta v$ case found in this analysis. It is easy to see that the required inclination changes are smaller than in the reference case previously shown in Fig. 6. In Fig. 8 (right), the asteroid Pallas is located just below the plane of the ecliptic, so the first $\Delta v$ is much smaller. The asteroid Pallas is in the position it would have been after 2 years of normal propagation, but the transfer times have been allowed to vary independently. The total trip time was still fixed at 7 years, regardless of the initial launch time that would be required to achieve the desired geometry.

\section{Conclusions}

With a complete set of series solutions available for every case of Lambert's Theorem, it is possible to optimize multiple-impulse missions to minimize total fuel requirements using analytical methods. Since the semi-major axis can be approximated as a polynomial in transfer time by truncating the infinite series solutions to a desired order, the transfer time itself may then appear explicitly in an expression for change in orbital velocities. The resulting 

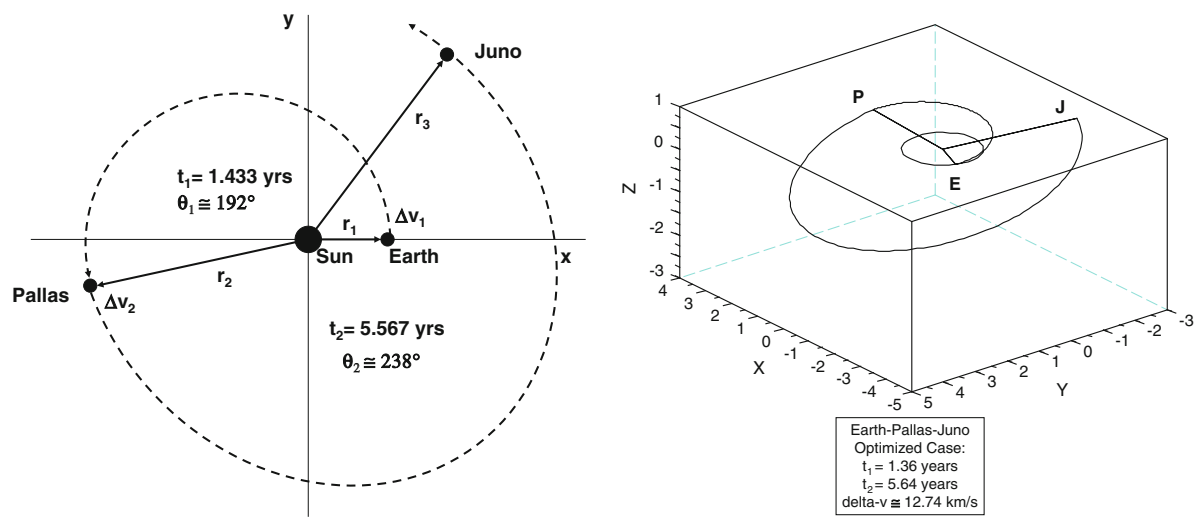

Fig. 8 Low delta-v case for Earth-Pallas_-Juno flyby mission: (left) in X-Y plane; (right) in 3D

algebraic expression may then be minimized over the complete set of transfer times. This approach was used successfully to reproduce a Hohmann transfer, a well known optimal solution for a circle-to-circle transfer with two maneuvers and a reference example from the literature of a modified Hohmann transfer with an associated plane change. The time series solutions of the Lambert problem were also used to minimize the fuel requirements for a three-dimensional example of an asteroid flyby mission with a total time constraint.

Open Access This article is distributed under the terms of the Creative Commons Attribution Noncommercial License which permits any noncommercial use, distribution, and reproduction in any medium, provided the original author(s) and source are credited.

\section{References}

Battin, R.H.: An Introduction to the Mathematics and Methods of Astrodynamics. AIAA Education Series, New York (1987)

Gooding, R.H.: A procedure for the solution of Lambert's orbital boundary-value problem. Celest. Mech. Dyn. Astron. 48, 145-165 (1990)

Lizia, P., Armellin, R., Lavagna, M.: Application of high order expansions of two-point boundary value problems to astrodynamics. Celest. Mech. Dyn. Astron. 102(4), 355-375 (2008)

Park, J., Lee, S., Choi, K.: Orbital maneuver using two-step sliding mode control. J. Astron. Space Sci. 15(1), 235-244 (1998)

Peterson, G.: Maneuver optimization for collision avoidance of LEO objects. In: Proceedings of 34th COSPAR Scientific Assembly, The Second World Space Congress, held 10-19 Oct, 2002 in Houston, TX, USA., meeting abstract

Thorne, J.D.: Lambert's theorem—a complete series solution. J. Astronaut. Sci. 52(4), 441-454 (2004)

Vallado, D.A.: Fundamentals of Astrodynamics and Applications, 3rd edn. Microcosm Press, California. Section 7.6.3. (2007) 